\title{
EINIGE RANDBEMERKUNGEN ZUM VERSTÄNDNIS DES ÄGYPTISCHEN WORTES „,W.t“ „ALTAR“
}

\author{
STEFAN BOJOWALD \\ Ägyptologisches Seminar der Universität Bonn \\ Regina-Pacis-Weg 7, 53113 Bonn, Deutschland \\ e-mail: stefan.bojowald@t-online.de
}

In the present paper a new explanation is suggested for the Egyptian word " $3 w . t$ " 'altar'. The old opinion stating a separate word with the meaning 'altar' is rejected. In this paper, the suggestion is put forward that it could be a secondary form of " $h 3 w . t$ ". It will be argued that the form came into existence by means of the "Lautwandel" between " 3 " and " $h$ ". Hitherto attested only in the Akkadian language, the "Lautwandel" between " $)$ " and " $h$ " can be pointed out in the Egyptian language for the first time.

Key words: Egyptian philology, new etymology, phonetic changes.

In der ägyptischen Sprache hat sich ein Wort „3w. $t^{\text {“ }}$ erhalten, das in den folgenden Zeilen noch einmal auf den Prüfstand gestellt werden soll. Das Wörterbuch (ErmanGrapow 1926, S. 5, 13) listet für den Begriff zwei Belege aus der 19. Dynastie auf. Das Wort hat nach den dortigen Angaben die Bedeutung „Altar" gehabt. Die Herausgeber gehen offenbar stillschweigend von einer eigenen Wurzel aus. In jedem Fall ist am genannten Ort nichts Gegenteiliges vermerkt worden.

Die Mitglieder des Berliner Wörterbuchprojekts (Reinecke 1993, S. 15) haben sich strikt an diese Linie gehalten, die in dieser Frage offenbar keinen Änderungsbedarf gesehen haben.

Das Digitale Zettelarchiv (DZA 20. 020.75) hat sich ebenfalls in diese Tradition gestellt, das mit der Bedeutung „Altar“ den Vorgängern gefolgt ist.

Das Wort kommt anscheinend nur an den beiden oben erwähnten Stellen vor. Die geringe Anzahl der Belege für ein Kulturwort wie „Altar“ will jedoch gerade in der tief religiös geprägten ägyptischen Gesellschaft nicht so richtig einleuchten. Die Beschränkung der Verwendungsdauer auf die 19. Dynastie stellt bei einem Wort, das in der Alltagssprache so oft benötigt wird, ebenfalls kein besonders realistisches 
Szenario dar. Der bisherige Ansatz soll hier daher kritisch hinterfragt werden. Der aktuelle Artikel wird stattdessen nach neuen Lösungswegen suchen.

In diesem Beitrag soll gezeigt werden, dass es sich bei ,3w.t" um eine Schreibung für , $h 3 w . t^{\prime \prime}$,Altar" handelt. Das Wort , $h 3 w . t$. ist von Anfang bis Ende der ägyptischen Sprachgeschichte zu beobachten (WB III, 226, 11-19) ${ }^{1}$. Das ägyptische Wort ,h3w.t" „Altar" wird von Orel und Stolbova (Orel-Stolbova 1995, no. 1308) auf eine gemeinhamito-semitische (afro-asiatische) Wurzel „* $h 3 w^{\text {" }}$ „Altar, Thron“ zurückgeführt. Das Wort ,h3ii.t" (WB III, 224, 13/14), „Altar" hat wohl zum gleichen Stamm gehört. In späterer Zeit ist auch die Schreibung von , $h 3 w . t$ " ohne auslautendes , $t$ " $\mathrm{zu}$ finden (WB III, 226, Belegschreibungen; el-Masry-Altenmüller-Thissen 2012, S. 102). Das Wort hat im demotischen die Form ,hwi“" angenommen (Erichsen 1954, S. 353). Das ägyptische ,h3w.t“ „Altar“ lebt im Koptischen als ,„унүє“ (Cerný 1976, S. 257; Vycichl 1983, S. 274; Westendorf 1965/1977, S. 334) weiter. Die Altarbezeichnung könnte auch als ägyptisches Fremdwort ins Akkadische gewandert sein. Die Auffassung wird so von Orel und Stolbova (Orel-Stolbova 1995, no. 1308) vertreten, nach deren Meinung das akkadische ,hawû" aus dem ägyptischen , $h 3 w . t$. hergeleitet werden kann. Die Gleichung ist auch von Militarev (Militarev 2007, S. 156-157) unter gewissen Einschränkungen gebilligt worden. Das Wort „hawû" hat ursprünglich eine Stoffart bezeichnet, die über Throne drapiert worden ist (CAD, 162-163). Das akkadische „hawû" selbst ist als Fremdwort ins Sumerische aufgenommen worden (Militarev 2007, S. 156).

Die Entstehung der Form ,3w. $t^{\prime \prime}$ aus , $h 3 w . t^{\prime \prime}$ ist theoretisch auf folgende Weise vorstellbar. Die Grundlage könnte der Lautwandel zwischen, ,3“ und , $h$ " gebildet haben, der die Assimilation von,,$h^{\prime \prime}$ an ,,$"$ " bewirkt hat. Der ägyptische Lautwandel zwischen ,";" und , $h^{\prime \prime}$ kommt hier offenbar zum allerersten Mal vor, so dass er noch nicht durch andere Quellen bestätigt worden ist. Die folgenden Beispiele könnten aber zu dessen approximativer Annahme berechtigen. Die Schreibung ,sf fh“ (Backes 2005, p. 190) für , ,sf $\}^{\text {“ }}$ könnte durchaus diesem Lautwandel entsprungen sein. Die Schreibung , hh h " für , $h 3 h^{\prime \prime}$, ,eilen“ könnte die gleiche Ursache haben, bei welcher der Lautwandel zur Assimilation von , ," an , $h$ " beigetragen hat. Die Stückzahl der Schreibung hat sich auf mindestens drei Beispiele belaufen. Das erste Fallbeispiel ist in: „,nb.t nriw h $\left\langle 3>h^{`} n\right.$ śbi.w“ (Kucharek 2010, S. 305) „Herrin des Schreckens mit schnellem Arm für die Rebellen ${ }^{2 ،}$ zu finden. Das zweite Fallbeispiel liegt in: ,hh $n=i$ mhỉ.t hntš = i im, tpi=i im r mrr=i“ (Jansen-Winkeln 1985, S. 141) „Der Nordwind ${ }^{3}$ eile zu mir, damit ich mich daran erfreue, damit ich davon atme nach meinem Belieben." vor. In der Sternenbezeichnung, ,hh nmt.t“ „Schnell an Schritt“ (Zandee 1947, S. 29) lässt

${ }^{1}$ Beachte die Schreibung von ,h3w.t" „Altar“ mit Krugdeterminativ bei Jansen-Winkeln (2007, S. 54 [1]), das es wohl vom ,h3wi“ - Gefäß bekommen hat, zu diesem Gefäßtyp vgl. Barns (1956, S. 20-31).

${ }^{2}$ Zum Wort „„śbi“ „Feind/Rebell“ vgl. Omar (2008, S. 185-192); zu „śbi““ „rebellieren“ im hamito-semitischen Kontext vgl. Diakonoff et al. (1997, S. 17).

${ }^{3}$ Beachte die fehlerhafte Schreibung , $m h\{n\}$ ii. $t$ “ für „,mhỉi.t“ „Nordwind“ bei Leitz (2011,

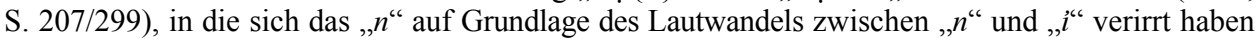
könnte; zu diesem Lautwandel vgl. Westendorf (1962, S. 29). 
sich das dritte Fallbeispiel entdecken. Die letzten Beispiele weisen zwar eine gewisse Ambivalenz auf, da der Schwund von ,";“ in der Wortmitte auch unter anderen lautlichen Voraussetzungen begegnet ist (Westendorf 1962, S. 9). Das dreimalige Auftreten der Erscheinung bei , $h 3 h^{“ *}$ spricht aber dafür, dass dahinter mehr als nur Zufall gestanden hat.

Der Beweis kann außerdem auf dem Umweg über die nächstverwandten Lautwandel erbracht werden. Der Lautwandel zwischen , $3 “$ und , $h “$ (Sethe 1899, S. 50) ist hierunter als erste Parallele hervorzuheben. Die ägyptische Sprache könnte ferner über den Lautwandel zwischen ,,$“$ und , $h$ "verfügt haben, für den an geeigneter Stelle eine eigene Untersuchung vorgelegt werden soll. Der Lautwandel zwischen , $i$ “" und , $h$ “ (Westendorf 1962, S. 36) könnte im hiesigen Kontext ebenfalls von Bedeutung sein. In diesem Zusammenhang braucht nur an den bestens bekannten Lautwandel zwischen , „'“" und „“" (Sethe 1899, S. 48; Edel 1955/1964, S. 59f; Westendorf 1962, S. 10f; Jasnow - Zauzich 2005, S. 89) erinnert zu werden. In Anbetracht der Lautwan-

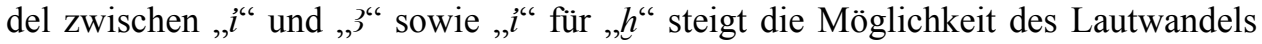
zwischen ,"'“ und , $h$ " proportional an. Das Hauptargument beruht jedoch auf der Tatsache, dass der Lautwandel zwischen , ," und , $h^{\prime \prime}$ im Repertoire der akkadischen Sprache vorhanden ist (von Soden 1995, 31 § 25c). Der Lautwandel konnte dort zwar nur in einem einzigen Beispiel definitiv nachgewiesen werden. Die Basis kann aber laut von Soden auch so als stabil gelten. Der Austausch zwischen ,"“" und , $h^{\prime \prime ~}$ tritt dagegen im Akkadischen auf schriftlicher Ebene sehr viel öfter auf (von Soden 1995, 31 \$25a). Das Akkadische hat zur gleichen hamito-semitischen (afro-asiatischen) Sprachfamilie wie das Ägyptische gehört, so dass es an der Legitimität des Vergleiches keine Zweifel geben kann.

Die Wahrscheinlichkeit nimmt somit in hohem Maße zu, dass der Lautwandel zwischen , ,“ und , $h^{\prime \prime}$ auch für die ägyptische Sprache angesetzt werden kann. Die große Seltenheit des Lautwandels spricht nicht gegen diese Einschätzung, da er - wie gesehen - im Akkadischen die gleiche Eigenschaft aufweist. Im Ergebnis läuft dies darauf hinaus, dass der Interpretation von ,,$w \cdot t^{\prime \prime}$,Altar“ als Schreibung für , $h 3 w . t^{*}$ „Altar“ so gut wie nichts mehr im Wege steht.

\section{Bibliographie}

Backes, B. (2005): Das altägyptische „Zweiwegebuch“. Studien zu den Sargtext - Sprüchen 1029 1130. Wiesbaden (Ägyptologische Abhandlungen 69).

Barns, J. W. B. (1956): Five Ramesseum Papyri. Oxford.

$\mathrm{CAD}=$ Oppenheim (1956).

Cerný, J. (1976): Coptic Etymological Dictionary. Cambridge-London-New York-Melbourne.

Diakonoff, Igor M. (Head of Team) - Belova, Anna G. - Militarev, Alexandre Ju. - Porkhomovsky,

Victor Ja. (1997): Historical Comparative Vocabulary of Afrasian. St. Petersburg Journal of African Studies 6, S. 12-35.

Edel, E. (1955/1964): Altägyptische Grammatik. Rom (Analecta Orientalia 34/39).

Erichsen, W. (1954): Demotisches Glossar. Kopenhagen.

Erman, Adolf-Grapow, Hermann (1926): Wörterbuch der Aegyptischen Sprache I. Berlin. 
Erman, Adolf-Grapow, Hermann (1982): Wörterbuch der aegyptischen Sprache III. Berlin.

Gardiner, A. H. (1916): Notes on the Story of Sinuhe. Paris.

Jansen-Winkeln, K. (1985): Ägyptische Biographien der 22. und 23. Dynastie. Teil 1: Übersetzung und Kommentar. Wiesbaden (Ägypten und Altes Testament 8).

Jansen-Winkeln, K. (2007): Drei Statueninschriften einer Familie aus frühptolemäischer Zeit. Studien zur Altägyptischen Kultur 36, pp. 49-79.

Jasnow, R. - Zauzich, K.-Th. (2005): The Ancient Egyptian Book of Thot. A Demotic Discourse on Knowledge and Pendant to the Classical Hermetica. Volume 1: Text. Wiesbaden.

Kucharek, A. (2010): Altägyptische Totenliturgien. Band 4: Die Klagelieder von Isis und Nephthys in Texten der griechisch-römischen Zeit. Heidelberg.

Leitz, Chr. (2011): Der Sarg des Panehemisis in Wien. Wiesbaden (Studien zur spätägyptischen Religion 3).

el-Masry, Y.-Altenmüller, H.-Thissen, H.-J. (2012): Das Synodaldekret von Alexandria aus dem Jahre 243 v. Chr. Hamburg (Studien zur Altägyptischen Kultur Beihefte 11).

Militarev, A. (2007): Akkadian-Egyptian Lexical Matches. In: Miller, Cynthia L. (ed.): Studies in Semitic and Afroasiatic Linguistics Presented to Gene B. Gragg. Chicago (Studies in Ancient Oriental Civilization 60), pp. 155-163.

Omar, M. (2008): Aufrührer, Rebellen, Widersacher, Untersuchungen zum Wortfeld „Feind“ im pharaonischen Ägypten. Ein lexikalisch-phraseologischer Beitrag. Wiesbaden (Ägypten und Altes Testament 74).

Oppenheim, A. L. (ed.) (1956): The Assyrian Dictionary. Volume 6, H. Glückstadt.

Orel, V. E. - Stolbova, O. V. (1995): Hamito-Semitic Etymological Dictionary: Materials for a Reconstruction. Leiden (Handbuch der Orientalistik 1: Naher und Mittlerer Osten 18).

Reinecke, W. F. (Hrsg.) (1993): Mitteilungen aus der Arbeit am Wörterbuch der ägyptischen Sprache. 2. Berlin, Brandenburgische Akademie der Wissenschaften.

Sethe, K. (1899): Das aegyptische Verbum im Altaegyptischen, Neuaegyptischen und Koptischen. Erster Band: Laut- und Stammeslehre. Hildesheim.

von Soden, W. (1995): Grundriss der akkadischen Grammatik. 3., ergänzte Auflage. Rom (Analecta Orientalia 33).

Vycichl, W. (1983): Dictionnaire étymologique de la langue copte. Leuven.

WB III = Erman - Grapow (1982).

Westendorf, W. (1962): Grammatik der medizinischen Texte. Berlin (Grundriss der Medizin der alten Ägypter VIII).

Westendorf, W. (1965/1977): Koptisches Handwörterbuch. Heidelberg.

Zandee, J. (1947): De Hymnen aan Amon van Papyrus Leiden I 350. Leiden (Oudheidkundige Mededelingen vit het Rijksmuseum van Oudheden, Nieuwe Reeks XXVIII). 